\title{
Autopercepción de la imagen corporal y prácticas para corregirla en adolescentes de una institución educativa, Lima-Perú
}

\author{
Self-perception of body image and practices to correct it in adolescents from an \\ educational institution, Lima, Peru
}

\author{
Raysa Emelin Enriquez Peralta, ${ }^{1, a}$, Margot Rosario Quintana Salinas ${ }^{1, b}$ \\ 'Escuela Académico Profesional de Nutrición, Facultad de Medicina, Universidad Nacional Mayor de San Marcos. Lima, Perú. \\ ${ }^{a}$ Licenciada en Nutrición; ${ }^{b}$ Docente del Departamento de Nutrición.
}

\begin{abstract}
Resumen
Introducción. Durante la adolescencia, etapa de cambios físicos y psíquicos, la imagen corporal se reviste de especial importancia; su insatisfacción puede contribuir a realizar prácticas inadecuadas. Objetivo. Identificar la autopercepción de imagen corporal y sus prácticas para corregirla. Diseño. Estudio mixto, cuanti-cualitativo. Lugar. Institución educativa privada, distrito Miraflores, Lima, Perú. Participantes. Adolescentes hombres y mujeres. Intervenciones. Se realizó mediciones antropométricas de peso y talla, encuesta de autopercepción de imagen corporal, y entrevistas a profundidad y grupales a 108 adolescentes hombres y mujeres para la fase cuantitativa, y 48 para la cualitativa. Principales medidas de resultados. Porcentaje de percepción equivocada de peso 0 talla. Percepción sobre su imagen corporal y prácticas para corregirla. Resultados. En el estudio, 36,1\% tenían percepción equivocada de su peso y $50,9 \%$ de su talla. Aunque más de la mitad de los entrevistados en la fase cualitativa tenía percepción equivocada del peso, mostraron aparente conformidad con su cuerpo; los que no lo estaban, manifestaban expresiones despectivas del mismo; ellos e incluso algunos conformes $(n=30)$ realizaban prácticas como dietas, actividad física y usaban medicamentos para bajar de peso 0 crecer; sus padres y amigos influian en la decisión de realizar dichas prácticas. Las participantes de once y doce años no mostraron interés en modificar su imagen corporal. Conclusiones. Casi un tercio de adolescentes tenía percepción equivocada de su peso, y la mitad de su talla. Los inconformes con su imagen corporal realizaban diferentes prácticas para corregirla.
\end{abstract}

Palabras clave: Adolescencia; Autopercepción; Dieta; Actividad física; Medicamentos.

\section{Abstract}

Introduction. Adolescence is a stage of physical and psychological changes; body image is of special importance and dissatisfaction with this can contribute to inappropriate practices. Objective. To identify self-perception of body image and practices adopted to change it. Design. Mixed quantitative and qualitative study. Setting. Private education institution, Miraflores district, Lima. Participants. Male and female adolescents. Interventions. In 108 boys and girls for the quantitative study, and 48 for the qualitative study, weight and height, body image self-perception, in-depth individual and group interviews were obtained. Main outcome measures. Percentage of misperception of weight or height. Perception of body image and practices applied to modify this. Results. In this study $36.1 \%$ had misperception of their weight and $50.9 \%$ of their height. Although most respondents in the qualitative study revealed misperception, they showed apparent conformity with their bodies; those who did not show conformity with their body, expressed scornful comments; these and others $(n=30)$ used diet, physical activity and medications to lose weight or to grow. Their parents and friends influenced their decisions. 11- and 12-year-old participants were not interested in modifying their body image. Conclusions. Nearly one third of adolescents had misperception of their weight and half of them in regard to their height. Those not accepting their body image practiced activities to correct it.

Keywords. Adolescence; Self-perception; Diet; Physical activity; Drugs.

An Fac med. 2016;77(2):117-22 / http://dx.doi.org/10.15381/anales.v77i2.11815

\section{INTRODUCCIÓN}

En el Perú, por mucho tiempo el tema hacia el cual se han centrado todos los esfuerzos en el ámbito nutricional ha sido la desnutrición infantil ${ }^{(1)}$. Se inició con la lucha contra la desnutrición aguda, peso bajo para la talla, cuya prevalencia actual en niños y niñas menores de 5 años es 0,6\%; continuó contra la desnutrición crónica, talla baja para la edad, problema que persiste al afec- tar a $14,6 \%$ de niños y niñas menores de 5 años ${ }^{(2)}$. Pero, de forma paralela, el sobrepeso y la obesidad están aumentando y afectan a $11 \%$ y $3,3 \%$ de adolescentes, respectivamente ${ }^{(3)}$, estableciéndose como un preocupante problema de salud pública, que requiere un abordaje especial de acuerdo a cada grupo etario.

La imagen corporal, esquema construido a partir de la historia personal y el momento histórico-social en el que se vive, se refiere a la configuración global formada por el conjunto de representaciones, percepciones, sentimientos y actitudes que el individuo ha elaborado con respecto a su cuerpo ${ }^{(4)}$; no es fija e inamovible, sino que cambia a lo largo de la vida e influye en la formación del autoconcepto y la autoestima del individuo, por lo que reviste una especial importancia en la adolescencia, momento en el que el aspecto 
físico se convierte en uno de los ejes de preocupación ${ }^{(5)}$.

El que no exista un correcto reconocimiento y aceptación de la imagen corporal puede contribuir a acciones inadecuadas, como la modificación de los hábitos alimentarios cuando realmente no es necesario o, en casos extremos, desarrollar trastornos de conducta alimentaria que podrían poner en riesgo la salud ${ }^{(4,6)}$. Así, los adolescentes realizarían prácticas que se podrían considerar correctivas de su peso, como dietas o actividad física no guiados por su peso corporal real sino por la percepción que tienen de su cuerpo y qué tan satisfechos están con él ${ }^{(6)}$. La especial preocupación por el cuerpo se puede iniciar en la adolescencia, pero también es probable que se presente en preadolescentes, una vez que ellos comienzan a tener conciencia de su imagen ${ }^{(7)}$.

En Chile, un estudio en estudiantes de Nutrición y Dietética muestra que casi la tercera parte de los encuestados consideraba tener un estado nutricional normal, casi la mitad se percibía con exceso de peso y $12,7 \%$ con peso bajo; mientras tanto, de acuerdo a su IMC, casi tres cuartas partes presentaban un estado nutricional normal, menos de la tercera parte exceso de peso y un $4 \%$ peso bajo ${ }^{(8)}$, lo que muestra que hay distorsión de la imagen corporal aún en estudiantes universitarios.

En Perú, un estudio en adolescentes de una institución educativa (IE) muestra que la mayoría realiza ejercicios como rutina diaria para bajar de peso; también se preocupan por reducir ciertas partes de su cuerpo. La pretensión de una imagen corporal perfecta produce distorsiones perceptivas y sitúa al adolescente en una experiencia de permanente insatisfacción corporal ${ }^{(9)}$.

Con el fin de lograr una perspectiva más holística y profunda del tema, se integró los enfoques de investigación cuantitativa y cualitativa; en lo último, el diseño utilizado fue el de teoría fundamentada, que tiene por objeto la identificación de procesos sociales bási- cos como punto central de la teoría; es útil para investigaciones en campos que conciernen a temas relacionados con la conducta humana ${ }^{(10)}$.

El objetivo de la presente investigación fue identificar la autopercepción de la imagen corporal y sus prácticas para corregirla.

\section{MÉTODOS}

Investigación de enfoque cuanticualitativo; en lo cuantitativo fue descriptivo, observacional y transversal y en lo cualitativo fue teoría fundamentada. El proyecto de estudio fue aprobado por el Comité de Investigación de la Escuela Académico Profesional de Nutrición de la Universidad Nacional Mayor de San Marcos. La población estuvo conformada por adolescentes hombres $(\mathrm{H})$ y mujeres $(\mathrm{M})$ de $1^{\circ}$ a $5^{\circ}$ de secundaria de una IE privada con administración religiosa, ubicada en el distrito de Miraflores y perteneciente a los niveles socioeconómicos A y B (11). Previas coordinaciones, se solicitó a los padres de familia su consentimiento informado para permitir la participación de sus hijos. Todos ellos aceptaron para la fase cuantitativa, pero tres no lo hicieron para la cualitativa; a todos los adolescentes se les solicitó su asentimiento informado. Se realizó un censo con los 108 adolescentes para aplicarles las técnicas de mediciones antropométricas de peso y talla ${ }^{(12)}$ con una balanza digital de pie con capacidad de 200 kilos, sensibilidad de $100 \mathrm{~g}$, marca SECA, y un tallímetro de madera portátil validado por el CENAN (Centro Nacional de Alimentación y Nutrición). Se aplicó una breve encuesta de autopercepción sobre imagen corporal validada por juicio de expertos y prueba piloto con 10 adolescentes de otra IE, que indagó si pensaban si su peso y talla era bajo, normal o alto, respectivamente, si su peso era adecuado para su talla y si su talla era adecuada para su edad; con ello se quería determinar si su autopercepción era correcta o equivocada. Después de registrar los datos de peso y talla y las respuestas de la encuesta en el pro- grama Microsoft office Excel 2010, los primeros se transfirieron al programa WHO Anthro Plus, para diagnosticar IMC para la edad (delgadez severa $<-3$ $\mathrm{DE}$, delgadez $<-2$ a $-3 \mathrm{DE}$, normal 1 a $-2 \mathrm{DE}$, sobrepeso $+1 \mathrm{a}+2 \mathrm{DE}$, obesidad $>2$ DE) y talla para la edad (talla baja severa $<-3 \mathrm{DE}$, talla baja $<-2$ a $-3 \mathrm{DE}$, normal +2 a $-2 \mathrm{DE}$, talla alta +2 DE). Luego, se aplicó la estadística descriptiva para hallar promedios, desviaciones estándar, porcentajes y frecuencias. Se comparó los diagnósticos nutricionales con las respuestas del cuestionario para establecer si la autopercepción de la imagen corporal era correcta o equivocada.

Para la fase cualitativa, la muestra estuvo conformada por 48 adolescentes elegidos intencionalmente, agrupados según percepción semejante de su peso o talla, independientemente de si fuera correcta o equivocada. Se aplicaron dos técnicas, entrevistas grupales ${ }^{(13)}$ y entrevistas a profundidad ${ }^{(13)}$, con guías semiestructuradas que abordaban los componentes perceptivo y cognitivoafectivo de la autopercepción de su imagen corporal y qué tipo de prácticas realizaban para corregirla. Las guías fueron revisadas por jueces expertos, seis nutricionistas y una psicóloga; después fueron adaptadas con una prueba de campo con tres adolescentes que no formaron parte de la muestra del estudio. Cada sesión fue en un ambiente propicio, grabada en audio y duraba entre 20 a 30 minutos. Las respuestas fueron transcritas a una hoja del programa Microsoft office Excel 2010, de tal manera que se construyó una matriz cuyas filas correspondían a las preguntas y las columnas a las respuestas de cada adolescente entrevistado, considerando sus datos de edad, sexo y cómo era la percepción de su peso y talla $(E=$ equivocada, $\mathrm{C}=$ correcta). Estas matrices fueron impresas en sábanas de $1 \mathrm{x}$ 4 metros, se les dio lectura junto con las notas de la bitácora de campo. En la bitácora de análisis, con codificación abierta se establecieron unidades de análisis. A las que compartían naturaleza, significado y características simi- 


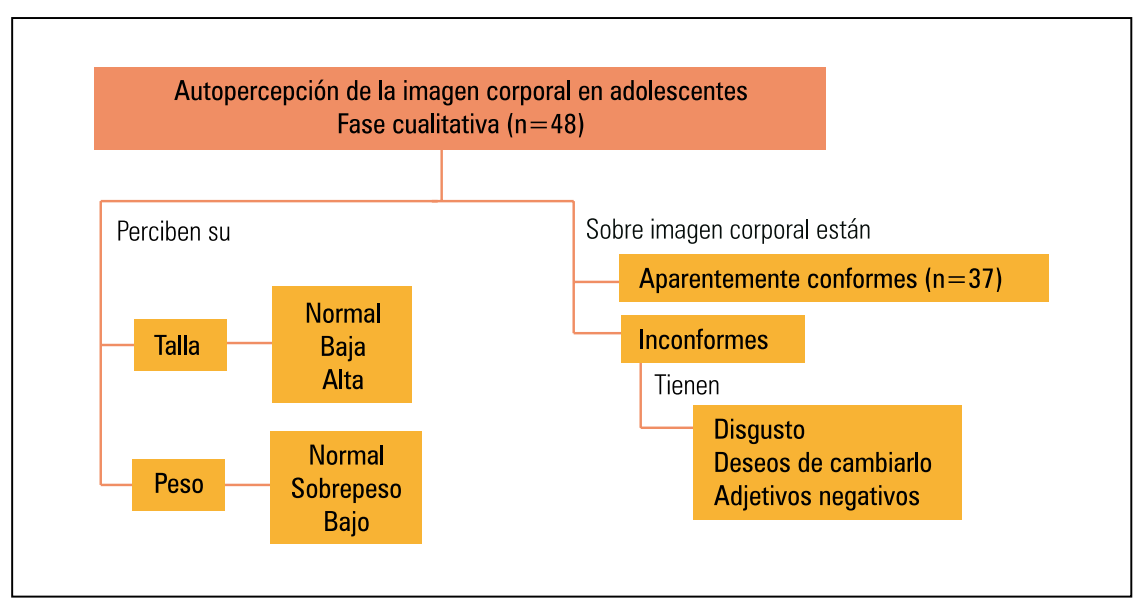

Figura 1. Autopercepción de la imagen corporal en adolescentes de una institución educativa de Lima.

lares se les asignó la misma categoría diferenciada por colores; luego, fue la codificación selectiva de aquellas categorías que guardaban relación con la categoría central, que explicaba y daba sentido a todos los demás datos y sus relaciones; y ya en la codificación teórica, se establecieron las relaciones o conexiones entre ellas ${ }^{(14)}$, lo que permitió elaborar figuras y tabla.

\section{RESULTADOS}

En la fase cuantitativa, los participantes tenían entre 11 y 17 años, con una media de 14 años $\pm 1,6 ; 58,3 \%$ eran mujeres. El 63\% tenía IMC/edad normal, $27,8 \%$ sobrepeso y $9,3 \%$ obesidad; todos tenían talla normal, excepto una adolescente; $54,8 \%$ percibía que su peso era normal $(\mathrm{M}=29,6 \%, \mathrm{H}=25 \%)$ al igual que su talla $48,1 \%(M=25,9 \%$, $\mathrm{H}=22,2 \%$; $31,5 \%$ percibía que su peso estaba aumentado $(\mathrm{M}=22,2 \%$, $\mathrm{H}=9,3 \%$ ), y $37 \%$ que su talla era baja $(\mathrm{M}=25 \%, \mathrm{H}=12 \%)$; $36,1 \%$ tenía percepción equivocada en cuanto a su peso $(M=20,4 \%, H=15,7 \%)$ y $50,9 \%$ en cuanto a su talla $(M=31,5 \%$, $\mathrm{H}=19,4 \%)$.

La mitad de participantes en la fase cualitativa era mujer $(n=24)$; la edad que predominó fue 15 años. La mayoría de entrevistados tenía una percepción equivocada tanto del peso $(\mathrm{PEP}=29)$ como de la talla $(\mathrm{PET}=34)$; esto fue mayor en mujeres ( $n=18$ y 20 , respectivamente).

Más de tres cuartos de los adolescentes expresaron conformidad con su imagen corporal $(n=37)$ "Yo creo que (mi cuerpo) está bien pero algunos me han dicho que estoy muy delgada pero yo pienso que estoy bien"; el resto estaba inconforme "No me gusta (mi cuerpo), porque soy cuadrada", "No me gusta, parezco una pelota". Más de la mitad de los que tenían percepción equivocada de su peso y talla $(n=19)$ manifestaron un disgusto evidente "No me gusta (mi cuerpo), es feo" (figura 1).

Tres cuartos de los adolescentes valoraba su cuerpo $(\mathrm{n}=36)$. "Lo valoro un montón, Dios me ha dado este cuerpo"; relacionaban esa valoración con el cuidarlo, evitar contraer alguna enfermedad, consumir drogas e incluso engordar. Mientras que otro grupo expresaba lo contrario $(\mathrm{n}=7)$ "Para mí no vale porque mi cuerpo es horrible". También hubo quienes manifestaban cuidarlo pero no se sentían conformes $(\mathrm{n}=5)$ "...lo cuido pero no es algo que yo quiero, si pudiera yo cambiaria mi cuerpo". Habían recibido comentarios negativos "...mi tío hace un tiempo me dijo que soy una pelota".

El ejemplo o la influencia de sus padres eran importantes para realizar alguna práctica "Mi padre siempre hace ejercicio, siempre intenta dietas y cosas asî"; también influían sus amigos.

Las actividades correctivas que manifestaron realizar para modificar su imagen corporal fueron actividad física $(n=36)$, hacer dieta $(n=23)$ y tomar medicamentos $(n=16)$; la razón común fue para bajar de peso (figura 2).

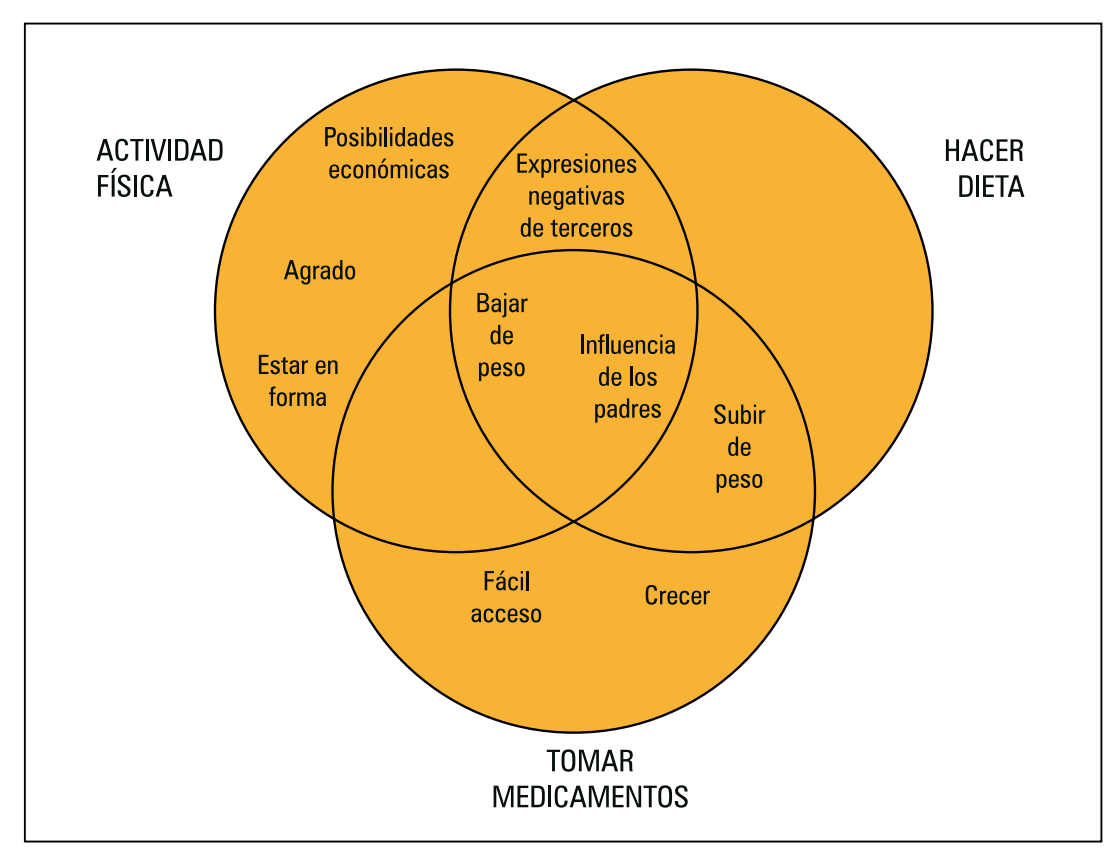

Figura 2. Razones para realizar prácticas correctivas de la imagen corporal en adolescentes. 
Declaraban seguir rutinas que obtenían de diversas fuentes, como entrenadores del gimnasio "...el entrenador me dice qué hacer", padres "... mi mamá y yo hicimos un horario para cosa (ejercicios) que tengo" e internet. Habían hecho o estaban haciendo dieta, para bajar o subir de peso; sin embargo, un grupo pequeño refirió no hacerla porque "...me da cosas (miedo) dejar de comer, me muero de hambre". Las dietas consistían en omitir comidas "No como en las noches y a veces en la mañana no como", comer saludable "Como saludable...en mi familia se come asî", y regímenes estrictos “... intenté dietas en las cuales comía una sola cosa al día”.

Las participantes de primero de secundaria (11 y 12 años) rechazaban la idea de hacer dieta e incluso la descartaban a futuro, mientras que las de mayor grado la habían realizado o al menos la habían considerado. Esas dietas provenían principalmente de internet; algunas habían visitado a especialistas, pero como no visualizaban el efecto deseado en corto tiempo, optaban por realizar otras acciones. Un grupo tomó pastillas, jarabes, laxantes y batidos para bajar o subir de peso, o para crecer.

Según ellos, otros adolescentes realizaban las siguientes prácticas para modificar su cuerpo: Ejercicios "Ir al gimnasio o practicar algún deporte", "Algunos me dicen que hacen abdominales", toma de pastillas "Mi amiga me ha dicho para ir al endocrinólogo que te da pastillas, algo parecido al té verde", hacer dietas, uso de inyecciones "quema grasa" y comer saludable.

En la tabla 1 se observa que, a mayor percepción equivocada de sus medidas corporales, los adolescentes participantes muestran mayor inconformidad con su aspecto y se incrementa la intensidad de las prácticas para corregirlas. Aquellos con percepción correcta solían contar con orientación del nutricionista o del entrenador del gimnasio para hacer dietas; mientras que los otros contaban con sus padres o internet.

\section{DISCUSIÓN}

En el presente estudio los porcentajes de sobrepeso y obesidad superaron a los reportados por la Encuesta Global de Salud Escolar (EGSE) del $2010^{(15)}$, realizada en estudiantes de educación secundaria de 50 instituciones educativas nacionales que presentaron 8 puntos porcentuales menos en sobrepeso y $3 \%$ de obesidad, en comparación al 9,3\% de este estudio realizado en una IE privada. Esto ratifica que el exceso de peso es problema de salud pública.

En una sociedad que glorifica la belleza, la juventud y la salud, no es extraño que aumente la preocupación por la apariencia física. Pero, la preocupación exagerada puede llegar a ser altamente perturbadora e incluso pro- blemática para muchas personas, sobre todo niños, niñas y adolescentes ${ }^{(16)}$. En el presente estudio, los adolescentes manifestaban sentirse preocupados por cómo se veían, y ya habían tomado la decisión de cambiar partes de su cuerpo que no creían adecuadas, aunque se notó que esa preocupación dependía de la edad. Así, las de 11 y 12 años no mostraban interés en ello e incluso rechazaban la idea de hacer dieta por considerarlo como una actividad incorrecta o difícil al relacionarla con dejar de comer ${ }^{(17)}$, tal vez porque el cambio físico se experimenta cuando el niño o niña va perdiendo su cuerpo infantil y eso suele suceder después de esa edad, con la pubertad, asociada a la ganancia fisiológica del peso que aumentaría la preocupación por la imagen corpo$\mathrm{ral}^{(18)}$.

La preocupación por su cuerpo no solo era por estética sino también por salud. Según Botelho ${ }^{(19)}$, las atenciones que los adolescentes brindan a su cuerpo van más allá de la presencia de algún grado de insatisfacción corporal; hay aspiración a una vida más saludable, por lo que varios siguen una alimentación saludable.

Mientras que otros estudios hallan que más de la mitad de adolescentes se percibe normal ${ }^{(20,21)}$, en el presente estudio fue menos de la mitad; en lo que se asemejan es en que las mujeres son quienes más se consideran con sobrepeso.

Tabla 1. Autopercepción de la imagen corporal según sea correcta o incorrecta y prácticas para corregirla.

\begin{tabular}{|c|c|c|c|}
\hline \multirow{2}{*}{ Subtemas } & \multicolumn{3}{|c|}{ Autopercepción de imagen corporal y prácticas para corregirla en adolescentes participantes, según percepción } \\
\hline & Correcta del peso y talla $(n=7)$ & Equivocada del peso o talla $(n=19)$ & Equivocada del peso y talla $(n=22)$ \\
\hline \multicolumn{4}{|l|}{ Autopercepción sobre } \\
\hline Cuerpo & Conformes; podría mejorar & Inconformes o resignados & Muy Inconformes \\
\hline Talla & Conformes & Quieren medir más & Quieren medir más \\
\hline Forma & Conformes & Conformes & No les gusta \\
\hline Valoración del cuerpo & Valoran & Valoran & La mitad no lo valora \\
\hline \multicolumn{4}{|l|}{ Prácticas } \\
\hline Dieta restrictiva & No & Mitad restringe comidas & Mitad omite comidas \\
\hline Actividad física & Por diversión, agrado, influenciados por padres & Para adelgazar, les gusta & Para adelgazar, crecer, estar sano \\
\hline Toma de medicamentos & No & Mitad los ha tomado & Tercera parte los ha tomado \\
\hline Usa medicina alternativa & No & Una mujer quisiera realizarla & Una mujer la realizó \\
\hline
\end{tabular}


Si bien la literatura informa que son las mujeres quienes con mayor frecuencia presentan insatisfacción corporal en la búsqueda del peso ideal (22), en el presente estudio también los hombres lo presentaban. La percepción equivocada, distorsionada en sentido negativo del propio cuerpo sería motivadora para realizar alguna práctica para corregirla. Míguez ${ }^{(23)}$ muestra que esa percepción distorsionada afecta a más de la mitad de su muestra y es más frecuente en hombres. En el presente estudio, casi la tercera parte de adolescentes tenía percepción equivocada de su peso y un poco más de la mitad de su talla; esto fue más frecuente en mujeres, cuyo aumento de grasa corporal determinan el cambio corporal. Bearman y cols. ${ }^{(24)}$ señalan que esta nueva estructura corporal se opone a lo establecido como un ideal de belleza para la mujer, que la expone a la presión por ser delgada, lo que contribuye a una menor satisfacción con la imagen corporal. Ante esta insatisfacción, los y las adolescentes del presente estudio realizaban prácticas para corregir su imagen corporal percibida, modificaban sus hábitos alimentarios, practicaban ejercicios y usaban medicamentos (pastillas- laxantes), principalmente para bajar de peso; también se mencionan dichas prácticas en otros estudios ${ }^{(25,26)}$.

La mayoría de las investigaciones sobre prácticas que los adolescentes realizan para bajar de peso han sido efectuadas en mujeres. En el presente estudio, al haber entrevistado a hombres y mujeres, se puede visualizar ciertas diferencias. Ellas son quienes presentan mayor recurrencia en prácticas de actividades para bajar de peso, como comer menor cantidad de comida u omitirlas, acciones ${ }^{(27)}$ que las pueden convertir en grupo de riesgo para padecer trastornos alimentarios (21). Los hombres no mencionan el uso de laxantes y medicamentos, a diferencia de otros brasileños ${ }^{(22,31)}$, quienes pueden consumir medicamentos para bajar de peso incluso sin prescripción médica ${ }^{(28)}$, lo cual es similar en las adolescentes del presente estudio, que ponen en evidencia la facilidad con la cual acceden a estos productos. Les era fácil dirigirse a una farmacia sin receta médica ni compañía de un adulto para obtenerlos; la televisión era la fuente de donde obtenían la idea de comprarlos. Los padres influyeron en esta decisión, sobre todo la madre; así, varias adolescentes decidieron tomar pastillas porque sus madres también lo hacían e, incluso en ocasiones, tomaban sus pastillas sin permiso. Esto demuestra que el entorno familiar y los padres transmiten mensajes en cuanto a apariencia y prácticas alimentarias, principalmente a través de su actitud y de su comportamiento como ejemplos ${ }^{(18,29,30,31)}$.

Es cierto que la actividad física practicada con regularidad y moderación es recomendable en cualquier etapa del desarrollo y que la adolescencia es una etapa para la adquisición de este hábito y su mantenimiento en la edad adulta. Sin embargo, en ocasiones esta actividad física se vuelve intensa y excesiva debido a una gran preocupación por el peso. La autopercepción de la condición física es determinante positivo del comportamiento físico-deportivo en hombres y mujeres ${ }^{(32)}$, lo cual se evidencia en el presente estudio cuando los entrevistados alegaban realizar actividad física principalmente para bajar de peso y estar en forma, aunque también habían quienes lo hacían por agrado a los deportes, diferente a lo hallado en un estudio cuantitativo ${ }^{(33)}$, en el que lo practicaban por salud.

La limitación del presente estudio fue que se hallaron pocos estudios con enfoque cualitativo referentes al tema que facilitara la discusión, aunque eso constituiría un aporte del presente, porque ofrece información de cómo los adolescentes percibían su propia imagen corporal además de conocer cómo afrontaban esa situación; esta información puede ser útil para dirigir intervenciones exitosas que consideren el componente emocional, punto clave en la adolescencia.

La fase cualitativa del presente estudio muestra que los y las adolescentes participantes, asistentes a una IE de NSE A y B, al querer cambiar su imagen corporal percibida realizaban prácticas como dietas, ejercicios y toma de medicamentos para bajar de peso o crecer. Estas prácticas fueron más intensas en aquellos que tenían percepción equivocada de su peso y talla, que son quienes suelen estar muy inconformes con su cuerpo, al punto de menospreciarlo. Las dietas fueron obtenidas principalmente de internet; las posibilidades económicas de los padres les permitían asistir a gimnasios e incluso armar uno en casa; y los medicamentos podían ser comprados por las madres, o ellos mismos adquirirlos en farmacias sin prescripción médica.

\section{REFERENCIAS BIBLIOGRÁFICAS}

1. Lanata CF. El problema del sobrepeso y la obesidad en el Perú: La urgencia de una política de salud pública para controlarla. Rev Peru Med Exp Salud Publica. 2012;29(3):299-300.

2. Instituto Nacional de Estadística e Informática. Encuesta Demográfica y de Salud Familiar. Lima; 2014.

3. Álvarez Dongo D, Sánchez Abanto J, Gómez Guizado G, Tarqui Mamani C. Sobrepeso y obesidad: prevalencia y determinantes sociales del exceso de peso en la población peruana (2009-2010). Rev Peru Med Exp Salud Pública. 2012;29(3):303-13.

4. Torres Guerrero J, Cepero González MdM, Torres Campos B. Relación entre la insatisfacción con la imagen corporal, autoestima, autoconcepto físico y la composición corporal en el alumnado de primer ciclo de educación secundaria de la ciudad de Jaén. 2010. Programa de doctorado: Actividad física para la educación en la sociedad del conocimiento.

5. Contreras Jordán OR, Gil Madrona P, Garcia López LM, Fernández Bustos JG, Pastor Vicedo JC. Incidencia de un programa de Educación Física en la percepción de la propia imagen corporal. Rev Educ. 2012;281-303.

6. Aventura Social. Promoção de competências e do capital social para um empreendedorismo com saúde na escola e na comunidade. Lisboa: Placebo; 2012.

7. Pallares Neila J, Baile Ayensa J. Estudio de la obesidad infantil y su relación con la imagen corporal en una muestra española. Psychol Av Discip. 2012;6(2):13-21.

8. Pino JL, López MA, Moreno AA, Pilar FTd. Percepción de la imagen corporal, del estado nutricional y de la composición corporal de estudiantes de nutrición y dietética de la Universidad del Mar, Talca, Chile. Rev Chil Nutr Vol. 2010;37(3):321-8.

9. Huarcaya C, Karina L. Relación entre la autopercepción de la imagen corporal y hábitos de alimentación en adolescentes del 5 to añode secundaria de la Institución Educativa Teresa Gonzáles de Fanning. 2008. 
10. Cuñat Giménez R. Aplicación de la Teoria Fundamentada (Grounded Theory) al estudio del proceso de creación de empresas. 2007. XX Congreso anual de Asociación Europea de Dirección y Economía de Empresa. Congreso Nacional.

11. Asociación Peruana de Empresas de Investigación de Mercados. Niveles Socio Económicos. 2013.

12. Instituto Nacional de Informática. Manual del Antropometrista. 2012. INEI-ENDES.

13. Pedraz A, Zarco J, Ramasco M, Palmar A Investigación cualitativa. Barcelona: Elsevier; 2014;60-71,87.

14. De la Torre G, Di Carlo E, Florido Santana A, Opazo Carvajal H, Ramírez Vega C, Rodríguez Herrero P, et al. Teoria Fundamentada o Grounded Theory. Universidad Autónoma de Madrid.

15. Ministerio de Salud. Encuesta Global de Salud Escolar-Resutados Perú 2010. Lima: MINSA; 2010.

16. World Health Organization. Nutrition in adolescence: issues and challenges for the health sector: issues in adolescent health and development. Suiza; 2005.

17. Pons D, Queralt A, Mars L, Garcia Merita M, Balaguer I. Estudio cualitativo de las conductas de salud en la primera adolescencia. Rev Latinoam Psicol. 2010;42(2):237-50.

18. Facchini M. La preocupación por el peso y la figura corporal en las niñas y adolescentes actuales: ¿de dónde proviene? Arch Argent Pediatr. 2006;104(4):345-50.

19. Bothelo da Costa SdM, Christa Machado MT. O corpo e a imagem corporal em adolescentes: perspectivas a partir do cuidado integral à saúde. Adolesc Saude. 2014;11(2):19-24.

20. Moreira Claro R, Siqueira Santos MA, Oliveira Campos M. Imagem corporal e atitudes extremas em relação ao peso em escolares brasileiros. Rev Bras Epidemiol. 2014;146-57.

21. López Atencio P, Molina Z, Rojas L. Influencia de género y la percepción de la imagen corporal en las conductas fluencia del género y la percepción de la imagen corporal en las conductas. An Venez Nutr. 2008:21(2):85-90

22. Moreira de Lima Pereira P, Costa do Carmo C, Carlos Cândido AP. Identificação da insatisfação corporal e comportamentos favoráveis ao desenvolvimento de transtornos alimentares em adolescentes de uma escola pública. Adolesc Saude. 2013;10(4):33-40.

23. Miguez Bernárdez M, De la Montaña Miguélez J, González Camero J, Gonzalez Rodríguez M. Concordancia entre la autopercepción de la imagen corporal y el estado nutricional en universitarios de Orense. Nutr Hosp. 2011;26:472-9.

24. Bearman S, Presnell K, Martínez E, Stice E. The skinny on body dissatisfaction: a longitudinal study of adolescent girls and boys. J Youth Adolesc. 2006;35(2):217-29.

25. Bojorquez I, Saucedo Molina TdJ, Juárez Garcia F, Unikel Santoncini C. Medio ambiente social y conductas alimentarias de riesgo: una exploración en mujeres adolescentes en México. Cad Saúde Pública. 2013;29(1):29-39.

26. Valois R, Zullig K, Huebner S, Drane W. Dieting behaviors, weight perceptions, and life satisfaction among public high school adolescents. Eating Disorders: The Journal of Treatment \& Prevention. 2003:11(4):271-88.

27. Quiles Marcos Y, Balaguer Solá I, Pamies Aubulat L, Quiles Sebastian MJ, Marzo Campos JC, Rodriguez Marín J. Eating habits, physical activity, consumption of substances and eating disorders in adolescents. The Spanish Journal of Psychology. 2011;14(2):712-23.

28. Ribeiro de Castro IR, Bertazzi Levy R, De Oliveira Cardoso L, Delboni dos Passos M, Vasconcelos Sardinha LM, Ferreira Tavares L, et al. Imagem corporal, estado nutricional e comportamento com relação ao peso entre adolescentes brasileiros. Ciência \& Saúde Coletiva. 2010;15(2):3099-4108.
29. McCabe M, Ricciardelli L Sociocultural influences on body image and body changes among adolescent boys and girls. J Soc Psychol. 2003;143(1):5-26.

30. Román V, Quintana M. Nivel de influencia de los medios de comunicación sobre la alimentación saludable en adolescentes de colegios públicos de un distrito de Lima. An Fac Med. 2010;71(3):185-9.

31. Rodríguez Ruis S, Díaz S, Ortega Roldán B, Mara JL, Delgado R, Férnandez Santaella MC. La insatisfacción corporal y la presión de la familia y del grupo de iguales como factores de riesgo para el desarrollo de los trastornos de la conducta alimentaria. Anuario de Psicologia Clínica y de la Salud. 2013:9:21-3.

32. Revuelta L, Esnaola I, Goñi A. El autoconcepto como determinante de la actividad físico-deportiva durante la adolescencia. Behav Psicol. 2013;21(3):581-601.

33. Castillo Fernández I, Balaguer Solá I. Dimensiones de los motivos de práctica deportiva de los adolescentes valencianos escolarizados. Educación Física y Deportes. 2001;63:22-9.

Artículo recibido el 11 de agosto de 2015 y aceptado para publicación el 21 de febrero de 2016.

Conflicto de intereses: Las autoras declaran que no existe conflicto de intereses en el presente manuscrito.

Correspondencia:

Raysa Emelin Enriquez Peralta.

Dirección: Mz. I 2 Lote 25 Urb. San Diego. Los Olivos. Lima, Perú.

Correo electrónico: raysa.nutricion@gmail.com 